\title{
Tumour stroma-derived Lipocalin-2 promotes breast cancer metastasis
}

Bilge Ören ${ }^{1}$, Jelena Urosevic ${ }^{2}$, Christina Mertens ${ }^{1}$, Javier Mora ${ }^{1,4}$, Marc Guiu ${ }^{2}$, Roger R. Gomis $^{2,3}$, Andreas Weigert ${ }^{1}$, Tobias Schmid ${ }^{1}$, Stephan Grein ${ }^{5}$, Bernhard Brüne ${ }^{1}$, Michaela Jung $^{1 *}$

${ }^{1}$ Institute of Biochemistry I, Goethe-University Frankfurt, Theodor-Stern-Kai 7, 60590 Frankfurt am Main, Germany.

${ }^{2}$ Oncology Program, Institute for Research in Biomedicine (IRB), 08028 Barcelona, Catalonia, Spain.

${ }^{3}$ Institució Catalana de Recerca I Estudis Avançats (ICREA), 08018 Barcelona, Catalonia, Spain.

${ }^{4}$ Faculty of Microbiology, University of Costa Rica, 2060 San José, Costa Rica.

${ }^{5}$ Goethe Center for Scientific Computing, Goethe-University Frankfurt, Kettenhofweg 139, 60325 Frankfurt am Main, Germany.

"Corresponding author: Dr. Michaela Jung, Goethe-University Frankfurt, Faculty of Medicine, Institute of Biochemistry I, Theodor-Stern-Kai 7, 60590 Frankfurt, Germany. Phone: +49-69-6301-6931 Fax: ＋49-69-6301-4203 Email: m.jung@biochem.unifrankfurt.de

This article has been accepted for publication and undergone full peer review but has not been through the copyediting, typesetting, pagination and proofreading process, which may lead to differences between this version and the Version of Record. Please cite this article as doi: $10.1002 /$ path.4724

This article is protected by copyright. All rights reserved. 


\begin{abstract}
Tumour cell-secreted factors skew infiltrating immune cells towards a tumour-supporting phenotype, expressing pro-tumourigenic mediators. However, the influence of lipocalin-2 (Lcn2) in the tumour microenvironment on the metastatic cascade is still not clearly defined. Here, we explored the role of stroma-derived, especially macrophage-released, Lcn2 in breast cancer progression. Knockdown studies and neutralizing antibody approaches showed that Lcn2 contributes to the early events of metastasis in vitro. The release of Lcn2 from macrophages induced an epithelial-to-mesenchymal transition program in MCF-7 breast cancer cells and enhanced local migration as well as invasion into the extracellular matrix using a 3D-spheroid model. Moreover, a global Lcn2deficiency attenuated breast cancer metastasis both in the MMTV-PyMT breast cancer model and in a xenograft model inoculating MCF-7 cells pre-treated with supernatants from wild type and Lcn2-knockdown macrophages. To dissect the role of stroma-derived Lcn2, we employed an orthotopic mammary tumour mouse model. Implantation of wild type PyMT tumour cells into Lcn2-lacking mice left primary mammary tumour formation unaltered, but specifically reduced tumour cell dissemination into the lung. We conclude that stroma-secreted Lcn2 promotes metastasis in vitro and in vivo, thereby contributing to tumour progression. Our study highlights the tumourigenic potential of stroma-released Lcn2 and suggests Lcn2 as putative therapeutic target.
\end{abstract}

Keywords: Lipocalin-2, tumour stroma, macrophage, metastasis, breast cancer, EMT

This article is protected by copyright. All rights reserved. 


\section{Introduction}

Lipocalin-2 (Lcn2) is a $25 \mathrm{kDa}$ glycoprotein of the lipocalin superfamiliy [1,2] that plays a pivotal role during bacterial infections [3], kidney regeneration [4], sepsis [5], and cancer [6]. Regarding tumour progression, several studies indicate that Lcn2 expression correlates with poor prognosis [7-9]. Additionally, Lcn2 serves as a prognostic and diagnostic marker, because elevated levels of Lcn2 are detected in the urine of cancer patients [9]. Lcn2 displays pleiotropic functions and promotes proliferation, survival, differentiation, and migration [10], thus rendering Lcn2 a putative mediator of tumour development. It was previously reported that Lcn2 promotes lung metastasis of murine breast cancer cells after injecting Lcn2-overexpressing 4T1 cells [11]. Lcn2 has been proposed to promote early events of tumour metastasis. On one side, tumour-supporting effects of Lcn2 can be explained by stabilizing gelatinase B (MMP-9) [12], thereby enhancing degradation of the extracellular matrix and tumour cell dissemination $[13,14]$. On the other side, Lcn2 induces epithelial-to-mesenchymal-transition (EMT). Specifically, overexpression of Lcn2 in MCF-7 breast cancer cells provokes EMT by reducing Ecadherin and increasing vimentin and fibronectin [9]. In contrast, the knockdown of Lcn2 in MDA-MB-231 breast cancer cells reverses EMT, associated with reduced tumour growth and metastasis [9]. In line with this, we recently described that Lcn2 conveys EMT characteristics to A375 melanoma cells, enhancing migration and invasion [15]. Moreover, the use of mouse mammary tumour models showed that $\mathrm{Lcn}^{-/-}$mice developed significantly fewer tumours, but differences in metastasis are still controversially discussed [16-18]. Importantly, the role of Lcn2 was mainly examined in tumour cells, whereas the possibility that Lcn2 is provided by tumour infiltrating immune cells, such as neutrophils or macrophages, was not taken into account so far.

Chronic inflammation and an impaired immune response provoke outgrowth of transformed cells and tumour progression [19]. Tumour-associated immune cells acquire a 
supportive phenotype to promote angiogenesis, metastasis, and tumour cell proliferation. Tumour-associated macrophages (TAM) are a prominent population of functionally polarized immune cells in the tumour microenvironment [20]. They infiltrate the majority of human tumours and are often linked to a poor prognosis [21]. TAM not only contribute to primary tumour growth, but also interact with tumour cells in the distinct phases of the metastatic route. There is strong evidence that migrating tumour cells co-localize with endothelial cells and macrophages in order to support metastatic spread [21-23]. The complex functional TAM phenotype is, at least in part, a response to tumour-derived components. We previously determined that apoptotic tumour cells activate the production and secretion of Lcn2 in macrophages with the subsequent polarization of these macrophages towards a pro-tumour phenotype [4]. Along these lines, we recently showed that macrophage-derived Lcn2 promotes proliferation of MCF-7 breast cancer cells [24]. Furthermore, inhibition of Lcn2 production in macrophages reduced renal regeneration when applying a macrophage-based cell therapy approach in a renal ischemia/reperfusion injury model, thereby substantiating the pro-proliferative and anti-inflammatory role of Lcn2 $[4,25]$.

Taking into account that Lcn2 conveys pro-proliferative, pro-regenerative, and antiinflammatory properties, we hypothesized that breast cancer progression might rely, at least in part, on the presence of Lcn2 in the tumour supportive microenvironment. We aimed at elucidating the role of macrophage-derived Lcn2 during the different stages of metastasis, including EMT, migration, and invasion, both in vitro and in vivo. 


\section{Materials and Methods}

\section{Cell culture}

The human breast cancer cell lines MCF-7 and MDA-MB-231, the hepatocellular carcinoma cell line $\mathrm{HUH7}$, and the lung carcinoma cell line A549 were cultured in Dulbecco's modified Eagle's medium (DMEM) with high glucose (Life Technologies, Darmstadt, Germany), supplemented with $100 \mathrm{U} / \mathrm{ml}$ penicillin (PAA Laboratories, Cölbe, Germany), $100 \mu \mathrm{g} / \mathrm{ml}$ streptomycin (PAA Laboratories), and 10\% fetal calf serum (FCS; PAA Laboratories). T47D human breast cancer cells were cultured in RPMI 1640 (Life Technologies), supplemented with $100 \mathrm{U} / \mathrm{ml}$ penicillin, $100 \mu \mathrm{g} / \mathrm{ml}$ streptomycin, and 10\% FCS. Tumour cells were cultivated in a humidified atmosphere with $5 \% \mathrm{CO}_{2}$ at $37{ }^{\circ} \mathrm{C}$ and passaged 3 times per week.

Primary human macrophages were isolated from human buffy coats (DRKBlutspendedienst Baden-Württemberg-Hessen, Frankfurt, Germany) as described previously [24].

Human pulmonary microvascular endothelial cells (HPMEC; PromoCell, Heidelberg, Germany) were cultured in Endothelial Cell Growth Medium (PromoCell) according to the manufacturers' instructions.

\section{Generation of macrophage-conditioned medium}

MDA-MB-231 cells were stimulated with $0.5 \mu \mathrm{g} / \mathrm{ml}$ staurosporine (LC Laboratories, Woburn, US) for $1 \mathrm{~h}$, washed with PBS, and incubated overnight in RPMI to generate apoptotic-conditioned medium (ACM). Primary human macrophages were stimulated with ACM for $6 \mathrm{~h}$ to induce Lcn2 production, washed with PBS, and cultured in RPMI overnight to generate macrophage-conditioned medium (MCM). To explore the role of Lcn2 in MCM, we applied a neutralizing antibody against Lcn2 $(3.5 \mu \mathrm{g} / \mathrm{ml}$; R\&D Systems MAB1757, 
Wiesbaden, Germany). An isotype matching IgG antibody (3.5 $\mu \mathrm{g} / \mathrm{ml}$; R\&D Systems 6001-A) was used as a control.

\section{Production of recombinant Lcn2}

Recombinant human Lipocalin-2 (LCN2) was produced by transformation of E. coli with a pGEX-4T-3-NGAL plasmid (kind gift from Dr. Anna Sola, IIBB Barcelona, Spain) expressing human LCN2 tagged to glutathione-S-transferase. LCN2 expression was initiated by supplementing isopropyl-ß-D-thiogalactopyranoside (Sigma-Aldrich, Steinheim, Germany) to the bacterial culture to activate the lac-operon. LCN was purified using ProCatch Glutathione Resin (Miltenyi Biotec, Bergisch Gladbach, Germany).

\section{Animal studies}

Experiments were conducted with approval of the Animal Care and Use Committee of: 1) Hessian review board (F144/12); and 2) the Institute for Research in Biomedicine (IRB Barcelona) and the Parc Científic de Barcelona (CEEA) (Catalan Government nDAAM: 7899).

\section{MMTV-PyMT breast cancer model}

C57BL/6 Lcn2 ${ }^{-/-}$mice (kind gift from Jack B. Cowland, University of Copenhagen, Denmark) were crossed into a C57BL/6 PyMT background. Tumour development was compared between wt (wild type; Lcn2 ${ }^{+/+}$) and Lcn2 $2^{-/-}$PyMT mice. Tumour growth was monitored to a maximal tumour diameter of $1.5 \mathrm{~cm}$ and tumour volume was calculated using the formula: Volume=length $\mathrm{x}$ width $^{2} \times 0.52$. After sacrifice, tumour and lung were isolated. Lung metastases were determined by Meyer's haemalum (Merck, Darmstadt, Germany) staining. The appearance of metastases was evaluated in 12 lung sections of different levels per mouse and the percentage of mice with lung nodules was calculated. Mice with an age of at least 20 weeks were taken into account. 


\section{Orthotopic breast cancer model}

Donors: female C57BL/6 wt PyMT and C57BL/6 Lcn-2 ${ }^{-/-}$PyMT mice. Recipients: female C57BL/6 Lcn-2 ${ }^{+/+}$and C57BL/6 Lcn2 ${ }^{-/-}$mice. The tumour from PyMT donor mice was isolated and dissociated with the Tumour Dissociation Kit (Miltenyi Biotec, Bergisch Gladbach, Germany). Cells were blocked with Fc Block Receptor Binding Inhibitor (eBioscience 130-092-575, Frankfurt, Germany) and stained with CD45 Vioblue (Miltenyi Biotec 130-102-430), CD326 PE (Miltenyi Biotec 130-096-448), and 7-AAD (BD Biosciences 559925). CD45/CD326 ${ }^{+}$living tumour cells were sorted using FACS Aria (BD Biosciences). Tumour cells $\left(5 \times 10^{5}\right)$ were implanted into the mammary gland (No.4) of recipient mice. The tumour was removed at a size of $1.5 \mathrm{~cm}$ and processed for flow cytometry analysis. Lungs were removed for immunofluorescence of PyMT (abcam Ab15085, Cambridge, UK) and Ki-67 (abcam Ab16667). The Opal ${ }^{\mathrm{TM}}$ 4-Color Fluorescent IHC Kit (PerkinElmer, Waltham, USA) was used according to the manufacturer's instructions. Co-localized PyMT- and Ki-67-double positive tumour cells were detected and quantified using the $\operatorname{Vectra}^{\circledR} 3$ automated quantitative pathology imaging system (PerkinElmer). The percentage of disseminated tumour cells was evaluated in three lung sections of different levels per mouse.

\section{$\underline{\text { Xenograft model }}$}

MCF-7 cells were pre-treated with MCM (scRNA MCM) and MCM from Lcn2-deficient macrophages (siLcn2 MCM) for 3 days. Subsequently, $3 \times 10^{6}$ tumour cells were resuspended in $25 \mu$ I PBS / $25 \mu$ l growth factor reduced Matrigel (Corning, New York, US) and injected into the mammary fat pad of BALB/c nude female mice. Each mouse received two plugs. Tumour growth was monitored to the size of $0.3 \mathrm{~cm}^{3}$. Oestrogen was provided by subcutaneous implantation of oestrogen pellets (90-day release) (Innovative research of America, USA) immediately after cell injections. Detection and quantification of 
disseminated tumour cells in the lung was performed using the IVIS-200 system [26]. Lung sections were stained with haematoxylin and eosin (H\&E). For immunohistochemistry, a rabbit monoclonal antibody to human oestrogen receptor (ER) alpha (Abcam, ab16660) and a mouse monoclonal antibody to human Ki-67 (Novocastra, NCL-L-Ki-67-MM1) were used.

\section{Small interfering RNA (siRNA) transfections}

HiPerFect transfection reagent (Qiagen, Hilden, Germany) was used according to the manufacturers' instructions. 50 nM SLC22A17 siRNA (Hs_SLC22A17_5 validated siRNA; Qiagen) was transfected into MCF-7 cells and 50 nM Lcn2 siRNA (Hs_LCN2_3 validated siRNA; Qiagen) was transfected into primary human macrophages. A non-targeting, scrambled siControl RNA (scRNA; Qiagen) was used.

\section{RNA extraction and Reverse Transcription-quantitative PCR (RT-qPCR)}

RNA extraction, reverse transcription and qPCR was performed as previously described [24]. The primers (human) used were: 18S: sense, 5'-GTA-ACC-CGT-TGA-ACC-CCA-TT3', antisense, 5'-CCA-TCC-AAT-CGG-TAG-TAG-CG-3'; E-cadherin (CDH1): sense, 5'-

TTC-CTC-CCA-ATA-CAT-CTC-CC-3', antisense, 5'-TTG-ATT-TTG-TAG-TCA-CCC-ACC3'; N-cadherin (CDH2): sense, 5'-CCT-GGA-GAC-ATT-GGG-GAC-TTC-A-3', antisense, 5'-GCC-ACT-GCC-TTC-ATA-GTC-AAA-CAC-3'; Lcn2 (LCN2): sense, 5'-TCA-CCC-TCTACG-GGA-GAA-CC-3', antisense, 5'-CCA-GCT-CCC-TCA-ATG-GTG-TT-3'; SLC22A17: sense, 5'-GCT-CTT-CGT-GGC-TCT-GGG-CAT-3', antisense, 5'-TGG-CAT-TGG-GAGGCT-GCT-3'.

\section{FACS}

Tumour cells were blocked with Fc Block Receptor Binding Inhibitor (eBioscience) and stained with CD45 APC-Cy7 (Biolegend, 103115), CD326 BV711 (BD Biosciences, 
563134), CDH1 FITC (eBioscience, 53-3249), CDH2 (abcam, Ab18203), Alexa Fluor 546 goat anti-rabbit IgG (Life Technologies, A11035), and were measured on a LSRFortessa flow cytometer.

\section{ELISA}

Detection of secreted Lcn2 was performed as described previously [24]. Lcn2 content was calculated per mg of total protein. The anti-Lcn2 (MAB1757), the secondary anti-rat IgG antibody (BAF005), Streptavidin-HRP and colour reagent were bought from R\&D Systems.

\section{Adhesion assays}

MCF-7 cells were stimulated with $1 \mu \mathrm{g} / \mathrm{ml}$ recombinant human Lcn2 or MCM for 4 days and marked with Cell Tracker green (Life Technologies). MCF-7 cells $\left(5 \times 10^{4}\right)$ were seeded into wells pre-coated with collagen I (10 $\mu \mathrm{g} / \mathrm{ml}$; BD Biosciences) or fibronectin (10 $\mu \mathrm{g} / \mathrm{ml}$; Sigma Aldrich), left for $2 \mathrm{~h}$, washed, then fixed with $4 \%$ PFA. Five pictures were taken for each group and from at least three independent experiments using triplicates and the number of attached cells was determined by ImageJ analysis (National Institutes of Health, Bethesda, US).

\section{Transendothelial migration assay}

MCF- 7 cells were stimulated with $1 \mu \mathrm{g} / \mathrm{ml}$ Lcn2 or MCM for 4 days and marked with Cell Tracker green. HPMEC $\left(1 \times 10^{5}\right)$ were seeded into fibronectin-coated Transwell inserts of a 24-well plate (Greiner Bio-One, Frickenhausen, Germany) and were grown to confluence. MCF-7 cells $\left(5 \times 10^{4}\right)$ were added into each insert. After $24 \mathrm{~h}$, cells were washed and fixed with 4\% PFA. Five fields per well were counted and the mean was calculated for each group. At least three independent experiments using triplicates were performed.

\section{Cell migration assay}

This article is protected by copyright. All rights reserved. 
MCF-7 cells were transfected either with siSLC22A17 or scrambled control siRNA (scRNA) and cell migration assay was performed as described previously [24]. Measurement of the scratch area was accomplished using Image J software. The $0 \mathrm{~h}$ value was normalized to the $24 \mathrm{~h}$ value of each group. The relative migration rate defines the mean value of at least three independent experiments using triplicates.

\section{Spheroid invasion assay}

MCF-7 spheroids were generated in 1.5\% agarose-coated 96-well-plates (Greiner BioOne). Spheroids were stimulated with scRNA MCM and siLcn2 MCM every 3 days for 2 weeks and subsequently embedded in a collagen I matrix for 7 days. 3 pictures were taken of each spheroid from each group (triplicates) from at least three independent experiments. Distance of invasion from the spheroid border into the collagen I matrix was measured using Axiovision software (Zeiss).

\section{Statistical analysis}

Each experiment was performed at least three times. The $p$ values were calculated using Student's $t$-test or one-way ANOVA, and considered significant at ${ }^{*} \mathrm{p}<0.05,{ }^{* *} \mathrm{p}<0.01$, ${ }^{* * *} p<0.001$ 


\section{Results}

\section{Lcn2 ablation delays tumour growth and metastasis of PyMT breast tumours}

We analysed the impact of Lcn2 in the spontaneous PyMT breast cancer model, where all female mice developed a tumour within 150 days in at least one mammary gland. We detected a significantly delay of tumour growth in Lcn2 ${ }^{-/}$PyMT mice starting at week $\sim 16$, whereas tumour growth in wt PyMT mice was already detected at week $~ 12$ (Fig. 1A). Accordingly, Lcn2 ${ }^{-/-}$PyMT mice reached the end point of $1.5 \mathrm{~cm}$ tumour diameter at later times (week 23), compared to wt PyMT mice that were sacrificed at week $\sim 19$ (Fig. 1B). In order to define the extent of overall tumour development, we chose 18 weeks, a timepoint when all mice showed tumour burden. Lcn2 ${ }^{-/-}$PyMT mice had smaller tumours and showed more mammary glands without tumour burden compared to wt PyMT mice (Fig. 1C), but the tumour diameter and number of glands with tumours did not significantly differ at the end point of $1.5 \mathrm{~cm}$ (Fig. 1D). Interestingly, we saw significant differences in the number of metastases-bearing mice comparing wt and $\mathrm{Lcn} 2^{-/-}$groups at sacrifice. The percentage of mice developing lung metastases was significantly lower in the Lcn ${ }^{-/-}$group compared to the wt group (Fig. 1E-F).

\section{Lcn2 induces metastatic spread in vitro}

We previously described that not only tumour cells, but also macrophages express Lcn2 after exposure to apoptotic tumour cell supernatants [4]. In the context of cancer, we reported that TAM-released Lcn2 supports tumour growth [24], but the impact on metastasis was not investigated so far. Therefore, we generated macrophage-conditioned medium containing Lcn2 (MCM) by stimulation of primary human macrophages with apoptotic tumour cell supernatants (ACM) and used in vitro lung metastasis assays to characterise the ability of macrophage-released Lcn2 to promote the metastatic tumour cell phenotype. Most interestingly, ACM-stimulated macrophages secreted significantly 
higher levels of Lcn2 compared to tumour cells (Fig. S1A and S1B). The attachment of tumour cells to extracellular matrix (ECM) components, including collagen I and fibronectin, is an essential step for tumour cell dissemination into the lung. Both MCM- and recombinant Lcn2 $(1 \mu \mathrm{g} / \mathrm{ml})$-stimulated cells significantly enhanced adhesion to both matrices. Quantification and representative pictures of adhesion to collagen I (Fig. 2A) and fibronectin (Fig. 2B) are displayed. Once tumour cells manage to adhere to the lung matrix, they migrate through the tight endothelial layer of pulmonary tissue, before a metastatic lesion can be established. To test the effect of Lcn2 on cancer cell migration across an endothelial layer, human pulmonary microvascular endothelial cells (HPMEC) were cultured in Transwell culture inserts. MCF-7 cells, pre-stimulated with MCM or recombinant human Lcn2 $(1 \mu \mathrm{g} / \mathrm{ml})$, passed more efficiently through this layer into the lower chamber of the Transwell (Fig. 2C). Thus, both MCM- and Lcn2-treatment enhanced the passage of tumour cells through a pulmonary, endothelial layer. As migration and invasion through the ECM are important events during metastasis, we examined the effect of macrophage-derived Lcn2 on tumour cell invasion, utilising a previously described 3D tumour spheroid model [27]. To verify Lcn2 as the responsible factor in the MCM inducing invasiveness of breast cancer cells, we established a transient knockdown of Lcn2 in primary human macrophages in order to generate Lcn2-deficient MCM (siLcn2 MCM). Control macrophages were treated with scRNA in order to generate supernatants containing Lcn2 (scRNA MCM) (Fig. S2). MCF-7 spheroids were stimulated with siLcn2 MCM, scRNA MCM, or left in normal growth medium (control) for ten days. Subsequently, spheroids were embedded into a collagen I matrix for additional seven days to allow invasion into the matrix. ScRNA MCM-treated spheroids showed significantly enhanced invasion into the collagen I matrix, whereas siLcn2 MCM-stimulated spheroids displayed reduced invasion (Fig. 2D).

\section{TAM-released Lcn2 facilitates lung colonization in vivo}

This article is protected by copyright. All rights reserved. 
As we had established that macrophage-derived Lcn2 induces metastatic spread in vitro, we wondered if these effects can be transferred to a breast cancer in vivo model. Therefore, we employed a xenograft model using MCF-7 cells that were pre-treated with scRNA MCM and siLcn2 MCM for 3 days. Our analysis of the tumour volume did not detect differences in tumour growth (Fig. 3A). Also, the weight of tumours at sacrifice was equivalent (Fig. 3B). Interestingly, the dissemination of implanted MCF-7 tumour cells to the lungs was significantly decreased by siLcn2 MCM-pre-treatment (Fig. 3C). Furthermore, histological analysis was performed in order to detect ER-positive proliferating (Ki-67-positive) tumour cell colonies (Fig. 3D). Representative pictures are shown for the co-localization of ER- and Ki-67-positive metastatic lesions. These data support the hypothesis that macrophage-derived Lcn2 increases the invasiveness of breast cancer cells, thereby facilitating metastatic spread.

\section{Macrophage-derived Lcn2 induces EMT through its specific receptor SLC22A17}

Since the higher migratory and invasive character of tumour cells is often associated with the transition from an epithelial to a mesenchymal state, the process of EMT plays an integral role in cancer metastasis. Therefore, we reasoned that macrophage-derived Lcn2 promotes EMT in breast cancer cells. MCM elicited a decrease in transcript abundance for the epithelial marker E-cadherin (CDH1) (Fig. 4A) and an increase of the mesenchymal marker N-cadherin (CDH2) (Fig. 4B) in human MCF-7 breast cancer cells. This effect was significantly attenuated by neutralizing Lcn2, while addition of the IgG control antibody was without effect. To exclude potential cell-specific effects, we verified changes in E-cadherin and N-cadherin mRNA abundance in response to MCM in T47D breast cancer cells, A549 lung carcinoma cells, and HUH7 hepatocellular cells (Fig. S3). In all cell lines tested, MCM decreased E-cadherin and increased $\mathrm{N}$-cadherin mRNA expression. These changes were significantly reversed by neutralizing Lcn2. Analysis of EMT-related transcription factors showed the potential involvement of ZEB1, whereas SNAI1 was not affected (Fig. S4). 
Neither Slug (SNAI2) nor Twist (TWIST1) transcripts could be detected. To explore the role of the specific Lcn2 receptor (SLC22A17) in transmitting EMT promoting effects on tumour cells, we performed a transient siRNA knockdown in MCF-7 cells (Fig. S5). Both, control (scRNA) and SLC22A17-downregulated tumour cells were stimulated with MCM for $24 \mathrm{~h}$ and the abundance of mRNA encoding the EMT markers E-cadherin (Fig. 4C) and Ncadherin (Fig. 4D) were measured by RT-qPCR. The previously observed impact of MCM on EMT marker expression was significantly reversed by the knockdown of SLC22A17. Since EMT correlates with increased migration rates, we explored if the knockdown of SLC22A17 affected tumour cell migration. Indeed, the knockdown of SLC22A17 impaired tumour cell migration (Fig. 4E). Quantification showed a significantly reduced migration rate in siSLC22A17 knockdown cells (Fig. 4F).

\section{Stroma-derived Lcn2 induces breast cancer cell dissemination into the lung}

In order to evaluate the impact of stroma-derived Lcn-2 on tumour growth and metastasis, we established an orthotopic breast cancer model. We used both wt and Lcn2 ${ }^{-/}$PyMT mice as tumour cell donors and $\mathrm{Lcn}^{+/+}$and $\mathrm{Lcn} 2^{-/-} \mathrm{C} 57 \mathrm{BL} / 6$ mice as recipients. A schematic picture of the model is shown in Figure 5A. Implantation of $\mathrm{Lcn}^{-/-}$tumour cells into $\mathrm{Lcn}^{+/+}$and $\mathrm{Lcn} 2^{-/-}$mice allowed us to analyse stroma-derived Lcn2 effects on tumour growth and metastasis. In addition, the comparison of implanted wt and Lcn2 ${ }^{-/-}$tumour cells allowed us to determine tumour cell-derived Lcn2 effects. We could not detect any differences in tumour development between $\mathrm{Lcn}^{+/+}$and $\mathrm{Lcn} 2^{-/-}$recipients, neither for Lcn2-competent donors (Fig. 5B), nor for Lcn2-deficient donors (Fig. 5C). Furthermore, the tumour weight at sacrifice did not differ significantly, suggesting that stroma-derived Lcn2 is not important for primary tumour growth (Fig. 5D-E). In order to detect lung metastases derived from implanted PyMT-positive tumour cells, we performed immunofluorescence using a PyMT specific antibody in combination with staining of proliferating tumour cells using Ki-67. Representative pictures show an efficient co-localization of PyMT- and Ki-67- 
positive tumour cells (Fig. 5F). Quantification of double-positive tumour cells showed that the injection of wt tumour cells into $\mathrm{Lcn}^{-/-}$recipient mice elicited a significant decrease in the number of disseminated Ki-67-positive PyMT tumour cells within the lung (Fig. 5G). However, implantation of Lcn2-deficient tumour cells resulted in a slight, but not significant reduction of the number of disseminated tumour cells in Lcn2 ${ }^{-/-}$recipient mice compared to wt recipients (Fig. $5 \mathrm{H}$ ). Remarkably, the $\mathrm{Lcn}^{-/-}$recipient showing decreased tumour cell dissemination, had a reduced number of $\mathrm{E}$-cadherin ${ }^{\text {low }} / \mathrm{N}$-cadherin ${ }^{\text {high }}$ tumour cells (Fig. 6A). The in vivo situation in the orthotopic model was corroborated in the PyMT model, where Lcn2-deficient PyMT mice revealed a reduced number of $\mathrm{E}$-cadherin ${ }^{\text {low }} / \mathrm{N}$ cadherin $^{\text {high }}$-tumour cells (Fig. 6B). Our results suggest that stroma-derived Lcn2 enhances metastatic spread by promoting different steps of the metastatic cascade, such as adhesion, transendothelial migration, invasion, and EMT (Fig. 6C).

This article is protected by copyright. All rights reserved. 


\section{Discussion}

The present study reveals a previously unknown function of stroma-derived Lcn2 on breast cancer metastasis. Evidently, macrophage-derived Lcn2 promotes tumour cell dissemination by inducing EMT, resulting not only in increased cancer cell motility and invasion, but also in enhanced transendothelial migration. A number of studies previously acknowledged a role of tumour-derived Lcn2 in tumour development and metastatic breast cancer progression, correlated with a poor prognosis $[9,16]$. The present study adds to the emerging role of Lcn2 for tumour progression. Interestingly, our data suggest a significantly higher expression of Lcn2 in ACM-treated macrophages than in tumour cells (Fig. S1). It was previously suggested that a threshold level of Lcn2 must to be achieved in tumour cells in order to promote EMT in Lcn2 overexpressing MCF-7 cells [9]. Therefore, it might be speculated that the local expression of Lcn2 from tumour-infiltrating macrophages adds to their pro-tumorigenic capacity. So far, most studies focused on the role of tumour cell-derived Lcn2, whereas the idea that Lcn2 might originate from tumourinfiltrating immune cells has so far not been appreciated. Shinriki et al. recently analysed the expression of Lcn2 in human oral squamous cell carcinoma (OSCC) [28]. They detected that poorly differentiated OSCC and reduced overall survival were particularly associated with Lcn2-expressing CD68 positive stromal immune cells rather than tumour cells, thus underscoring the importance of monocyte-derived Lcn2. These findings point to a role of stroma-derived Lcn2 as a previously unrecognized factor in tumour progression. However, we previously showed that macrophage-released Lcn2 enhanced the proliferative capacity of MCF-7 breast cancer cells in vitro [24]. To prove the impact of macrophage-derived Lcn2 on breast cancer progression in vivo, we used a xenograft model involving MCF-7 cells pre-treated with macrophage supernatants and an orthotopic mammary tumour model. Neither the xenograft (Fig. 3A-B), nor the orthotopic model (5BE) displayed differences in primary tumour growth. In the present study, we also checked 
for tumour growth, both in a 3D tumour spheroid model and in 2D cell culture, but failed to detect differences in primary growth characteristics in the complex 3D system, whereas the 2D cultured responded as described before [24]. Corroborating our data, it was recently published that supernatants of macrophages co-cultured with apoptotic tumour cells promote the aggressiveness of MCF-7 cells by enhancing tumour growth and metastasis [29].

There is growing evidence that Lcn2 promotes the development of malignant tumour cell phenotypes by inducing EMT $[9,15]$, an essential early step towards tumour metastasis. The transition from an epithelial into a mesenchymal cell is characterised by the loss of epithelial cell polarity, the loss of cell-cell contacts, and increased cell motility. An important characteristic of EMT is the cadherin switch, characterised by decreased Ecadherin, but increased $\mathrm{N}$-cadherin expression. E-cadherin promotes cell-cell adhesion through homophilic interactions between E-cadherin proteins on adjacent cells, thus forming adherens junctions [30]. In contrast, N-cadherin causes tumour progression and invasion. This is facilitated through interactions with fibroblast growth factor receptor 1 , activating the mitogen-activated protein kinase (MAPK)/extracellular signal-regulated kinase (ERK) pathway to enhance MMP-9 expression [30,31]. Interestingly, Lcn2 was shown to support tumour progression by stabilizing MMP-9, thereby facilitating ECM degradation [12,14]. Apparently, tumour cell-derived Lcn2 as well as stroma-derived Lcn2 might be important players during the metastatic spread. However, our orthotopic tumour model suggests that predominantly stroma-derived Lcn2 induces metastasis by promoting tumour cell dissemination into the lung. Therefore we questioned whether this results from the induction of EMT in tumour cells. In fact, we showed that macrophage-derived Lcn2 induces EMT in MCF-7 breast cancer cells by reducing the epithelial marker E-cadherin and inducing the mesenchymal marker N-cadherin (Fig. 4A-D). Importantly, we observed a more robust and consistent Lcn2-induced expression of $\mathrm{N}$-cadherin rather than repression 
of E-cadherin in vitro. However, it is important to note that EMT usually is not a complete transition but rather a transient and reversible process, since many tumours might also coexpress both cadherins, illustrating thereby their plasticity [32]. Nevertheless, it is speculated that a proportion of fully acquired EMT-cells are required for effective invasion through the ECM, thereby opening the way for non- or transient-EMT-cells to enter the bloodstream and to spread into distant organs.

In summary, we propose that stroma-derived Lcn2 enhances the malignant characteristics of breast cancer cells. Our studies underscore the significance of stromaderived Lcn2 on tumour cell dissemination and metastatic growth and offer new therapeutic perspectives. Nevertheless, further research is needed to define the diverse biological effects of Lcn2 within the tumour microenvironment.

This article is protected by copyright. All rights reserved. 


\section{Acknowledgements}

We thank Jack B. Cowland for kindly providing the Lcn2 ${ }^{-/-}$mice (National University Hospital, University of Copenhagen, Copenhagen, Denmark) and Dr. Anna Sola for kindly sharing the pGEX-4T-3-NGAL plasmid. The study was supported by the Fritz Thyssen Stiftung (grant number: Az.10.12.2.156, awarded to MJ), Goethe-University (Focus Line $B$, awarded to $M J$ ), and the Monika Kutzner Stiftung (awarded to MJ). RRG laboratory is supported by the Spanish Ministry of Science and Innovation grant SAF2013-46196 and the Generalitat de Catalunya AGAUR 2014-SGR grant 535. Further support came from the Deutsche Krebshilfe (111578 to BB).

\section{Author contribution statement}

The authors contributed in the following way: BÖ: data acquisition, analysis, and interpretation, and writing of the manuscript; JU: data acquisition, analysis, and interpretation; CM, JM, RRG, AW: data analysis and interpretation; MG: data acquisition and analysis; TS, BB: data interpretation and writing of the manuscript; SG: generation of figures by bioinformatics tools; MJ: study design and supervision, data acquisition and analysis, and writing of the manuscript. All authors read and agreed on the final manuscript. 


\section{References}

1. Kjeldsen $\mathrm{L}$, Johnsen $A H$, Sengelov $H$, et al. Isolation and primary structure of NGAL, a novel protein associated with human neutrophil gelatinase. J Biol Chem 1993; 268: 10425-10432.

2. Virzì GM, Clementi A, Cal M de, et al. Genomics and biological activity of neutrophil gelatinase-associated lipocalin in several clinical settings. Blood Purif 2013; 35: 139_ 143.

3. Flo $\mathrm{TH}$, Smith $\mathrm{KD}$, Sato $\mathrm{S}$, et al. Lipocalin 2 mediates an innate immune response to bacterial infection by sequestrating iron. Nature 2004; 432: 917-921.

4. Sola $A$, Weigert $A$, Jung $M$, et al. Sphingosine-1-phosphate signalling induces the production of Lcn-2 by macrophages to promote kidney regeneration. J Pathol 2011; 225: 597-608.

5. Srinivasan G, Aitken JD, Zhang B, et al. Lipocalin 2 deficiency dysregulates iron homeostasis and exacerbates endotoxin-induced sepsis. J Immunol 2012; 189: 1911-1919.

6. Rodvold JJ, Mahadevan NR, Zanetti M. Lipocalin 2 in cancer: When good immunity goes bad. Cancer Lett 2012; 316: 132-138.

7. Leng $\mathrm{X}, \mathrm{Wu} \mathrm{Y}$, Arlinghaus RB. Relationships of lipocalin 2 with breast tumorigenesis and metastasis. J Cell Physiol 2011; 226: 309-314.

8. Yang J, Moses MA. Lipocalin 2: A multifaceted modulator of human cancer. Cell Cycle 2009; 8: 2347-2352.

9. Yang J, Bielenberg DR, Rodig SJ, et al. Lipocalin 2 promotes breast cancer progression. Proc Natl Acad Sci U S A 2009; 106: 3913-3918. 
10. Chakraborty S, Kaur S, Guha S, et al. The multifaceted roles of neutrophil gelatinase associated lipocalin (NGAL) in inflammation and cancer. Biochim Biophys Acta 2012; 1826: $129-169$.

11. Shi H, Gu Y, Yang J, et al. Lipocalin 2 promotes lung metastasis of murine breast cancer cells. J Exp Clin Cancer Res 2008; 27: 83.

12. Yan L, Borregaard N, Kjeldsen L, et al. The high molecular weight urinary matrix metalloproteinase (MMP) activity is a complex of gelatinase B/MMP-9 and neutrophil gelatinase-associated lipocalin (NGAL). Modulation of MMP-9 activity by NGAL. J Biol Chem 2001; 276: 37258-37265.

13. Deryugina EI, Quigley JP. Matrix metalloproteinases and tumor metastasis. Cancer Metastasis Rev 2006; 25: 9-34.

14. Kubben FJGM, Sier CFM, Hawinkels LJAC, et al. Clinical evidence for a protective role of lipocalin-2 against MMP-9 autodegradation and the impact for gastric cancer. Eur J Cancer 2007; 43: 1869-1876.

15. Gheorgheosu D, Jung M, Oren B, et al. Betulinic acid suppresses NGAL-induced epithelial-to-mesenchymal transition in melanoma. Biol Chem 2013; 394: 773-781.

16. Leng $\mathrm{X}$, Ding $\mathrm{T}$, Lin $\mathrm{H}$, et al. Inhibition of lipocalin 2 impairs breast tumorigenesis and metastasis. Cancer Res 2009; 69: 8579-8584.

17. Berger T, Cheung CC, Elia AJ, et al. Disruption of the Lcn2 gene in mice suppresses primary mammary tumor formation but does not decrease lung metastasis. Proc Natl Acad Sci U S A 2010; 107: 2995-3000.

18. Cramer EP, Glenthoj $A$, Hager $M$, et al. No effect of NGAL/lipocalin-2 on aggressiveness of cancer in the MMTV-PyMT/FVB/N mouse model for breast cancer. PloS One 2012; 7: e39646. 
19. Quail DF, Joyce JA. Microenvironmental regulation of tumor progression and metastasis. Nat Med 2013; 19: 1423-1437.

20. Jung $M$, Mertens $C$, Brüne $B$. Macrophage iron homeostasis and polarization in the context of cancer. Immunobiology 2015; 220: 295-304.

21. Qian BZ, Pollard JW. Macrophage diversity enhances tumor progression and metastasis. Cell 2010; 141: 39-51.

22. Pollard JW. Trophic macrophages in development and disease. Nat Rev Immunol 2009; 9: 259-270.

23. Rohan TE, Xue X, Lin $\mathrm{H}$, et al. Tumor Microenvironment of Metastasis and Risk of Distant Metastasis of Breast Cancer. J Natl Cancer Inst 2014; 106: dju136.

24. Jung $M$, Weigert $A$, Tausendschon $M$, et al. Interleukin-10-induced neutrophil gelatinase-associated lipocalin production in macrophages with consequences for tumor growth. Mol Cell Biol 2012; 32: 3938-3948.

25. Jung $M$, Sola A, Hughes J, et al. Infusion of IL-10-expressing cells protects against renal ischemia through induction of lipocalin-2. Kidney Int 2012; 81: 969-982.

26. Minn AJ, Gupta GP, Siegel PM, et al. Genes that mediate breast cancer metastasis to lung. Nature 2005; 436: 518-524.

27. Werno C, Menrad H, Weigert A, et al. Knockout of HIF-1a in tumor-associated macrophages enhances M2 polarization and attenuates their pro-angiogenic responses. Carcinogenesis 2010; 31: 1863-1872.

28. Shinriki S, Jono $\mathrm{H}$, Ueda $\mathrm{M}$, et al. Stromal expression of neutrophil gelatinaseassociated lipocalin correlates with poor differentiation and adverse prognosis in oral squamous cell carcinoma. Histopathology 2014; 64: 356-364. 
29. Zhou N, Zhang $\mathrm{Y}$, Zhang $\mathrm{X}$, et al. Exposure of tumor-associated macrophages to apoptotic MCF-7 cells promotes breast cancer growth and metastasis. Int J Mol Sci 2015; 16: 11966-11982.

30. Yilmaz M, Christofori G. EMT, the cytoskeleton, and cancer cell invasion. Cancer Metastasis Rev 2009; 28: 15-33.

31. Hazan RB. Exogenous Expression of N-Cadherin in Breast Cancer Cells Induces Cell Migration, Invasion, and Metastasis. J Cell Biol 2000; 148: 779-790.

32. Christiansen JJ, Rajasekaran AK. Reassessing epithelial to mesenchymal transition as a prerequisite for carcinoma invasion and metastasis. Cancer Res 2006; 66: 8319-8326.

This article is protected by copyright. All rights reserved. 
Supplementary Material on the Internet

\section{Supplementary figure legends}

Figure S1. Release of Lcn2 from macrophages and tumour cells

Figure S2. Knockdown efficiency of Lcn2 in primary human macrophages

Figure S3. Macrophage-derived Lcn2 induces EMT in various cancer cell lines

Figure S4. EMT-associated transcription factors

Figure S5. Knockdown of the Lcn2 receptor SLC22A17 in MCF-7 breast cancer cells 
Figure legends
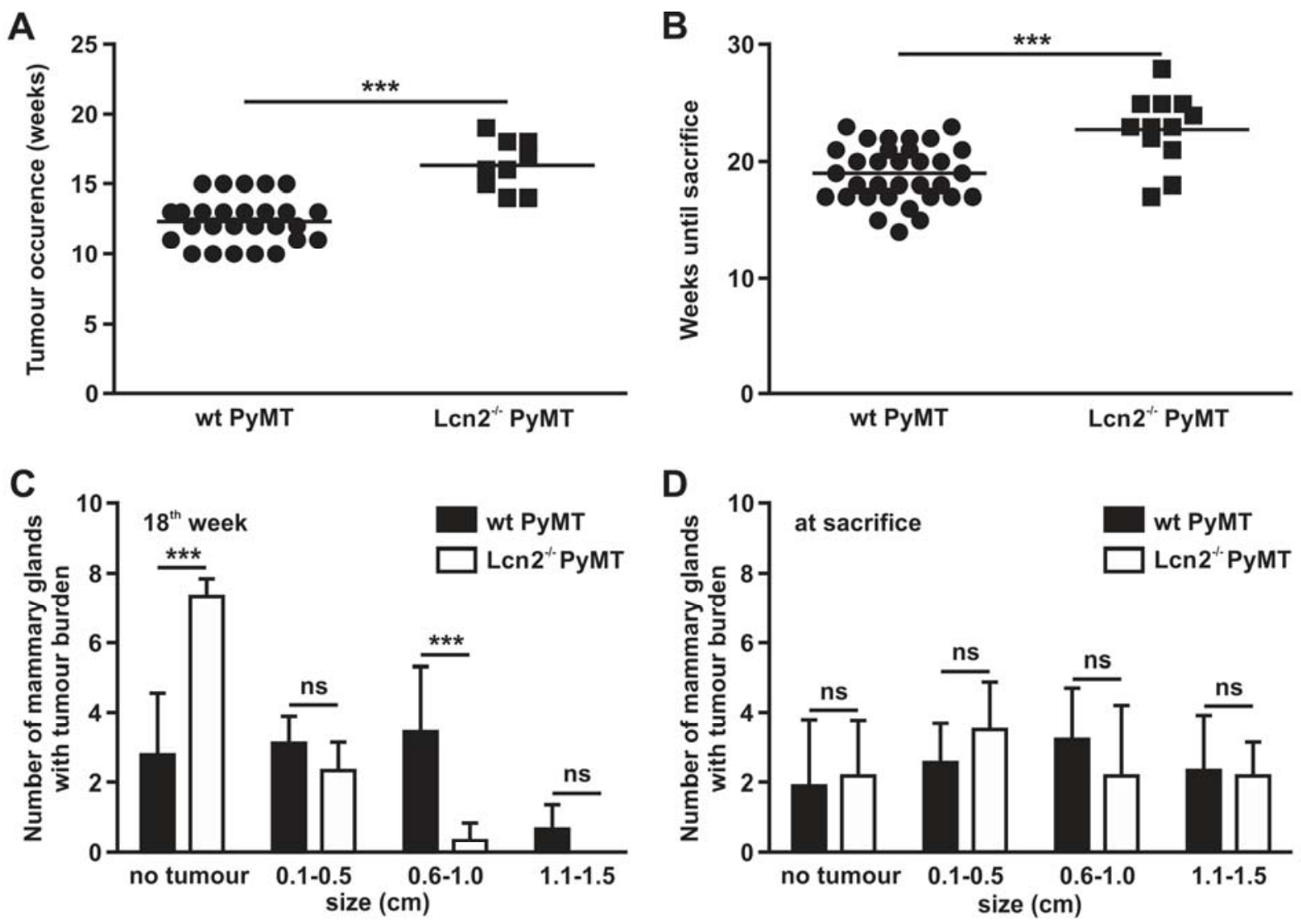

E

$\mathbf{F}$
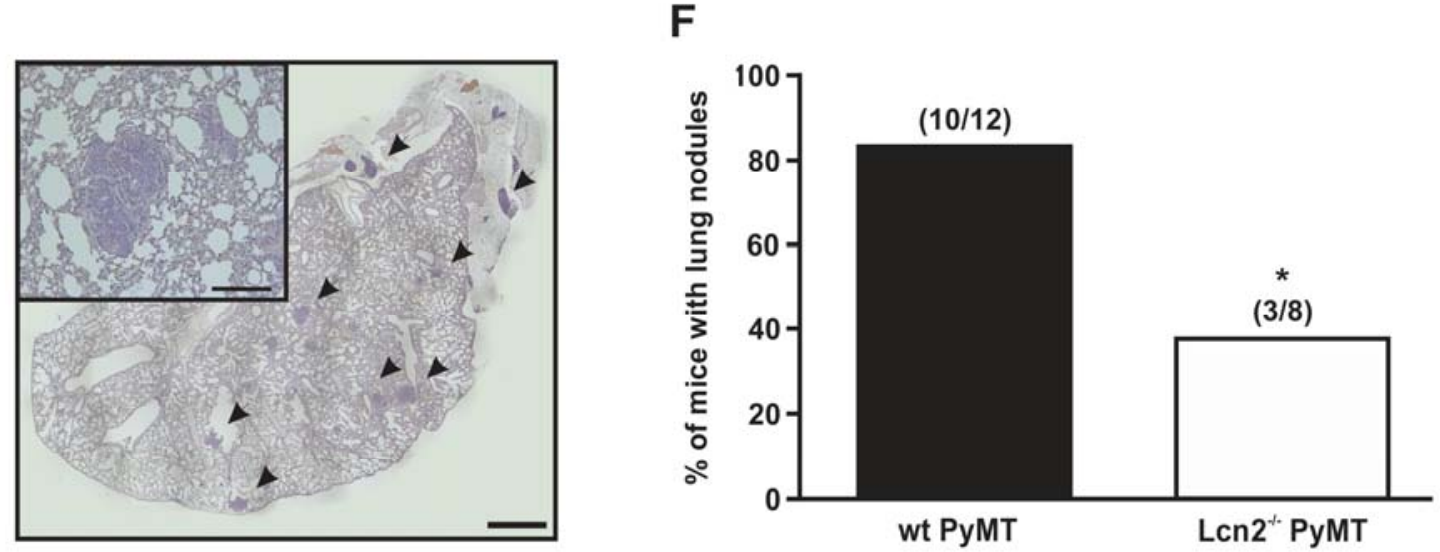

Fig. 1: Len2 ablation delays tumour growth and reduces lung metastasis of PyMT breast tumours 
(A) Tumour occurrence in wt PyMT $(n=27)$ and Lcn2 $2^{-/-}$PyMT $(n=9)$ mice. (B) Time until sacrifice displayed for wt PyMT $(n=33)$ and Lcn2 $2^{-1-}$ PyMT $(n=12)$ mice. (C) Number of mammary glands with tumour burden and tumour size was assessed by palpation of wt PyMT $(n=9)$ and Lcn2 $2^{-/-}$PyMT $(n=6)$ mice at the age of 18 weeks and $(D)$ at sacrifice. $(E-F)$ After sacrificing, lung sections were stained with Meyer's haemalum. (E) Representative whole lung section (scale bar: $1000 \mu \mathrm{m}$ ) and magnification of a metastatic lesion (scale bar: $200 \mu \mathrm{m}$ ). Arrows point to metastatic nodules. (F) The percentage of mice with metastatic lesions were counted in wt PyMT $(n=12)$ and Lcn2 ${ }^{-/}$PyMT $(n=8)$ mice. Data are means \pm S.D., ${ }^{*} p<0.05$ vs. controls, ${ }^{* * *} p<0.001$ vs. controls. 
A

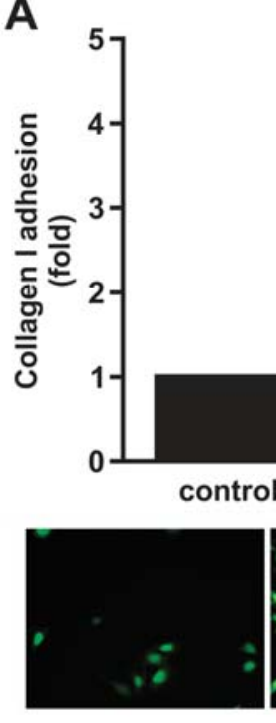

control

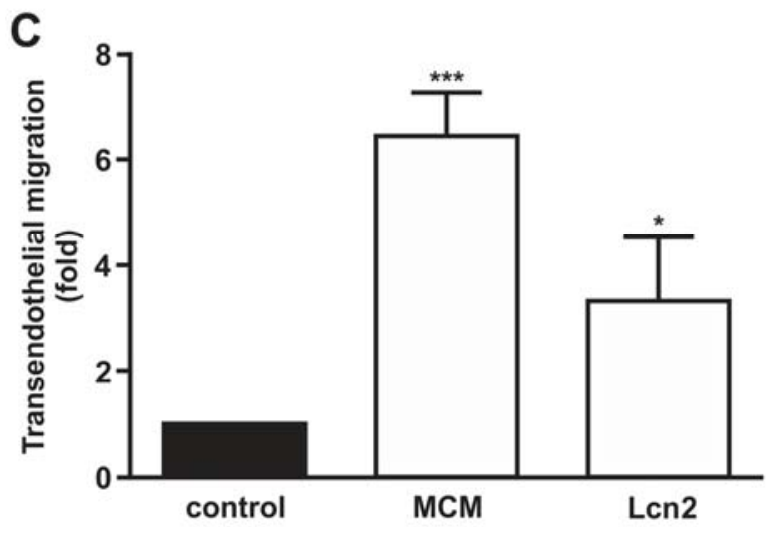

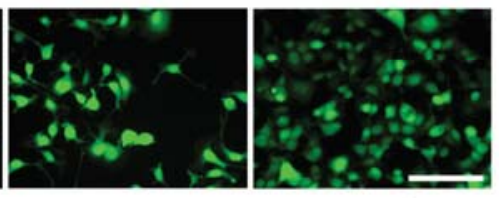

MCM

Lcn2
B
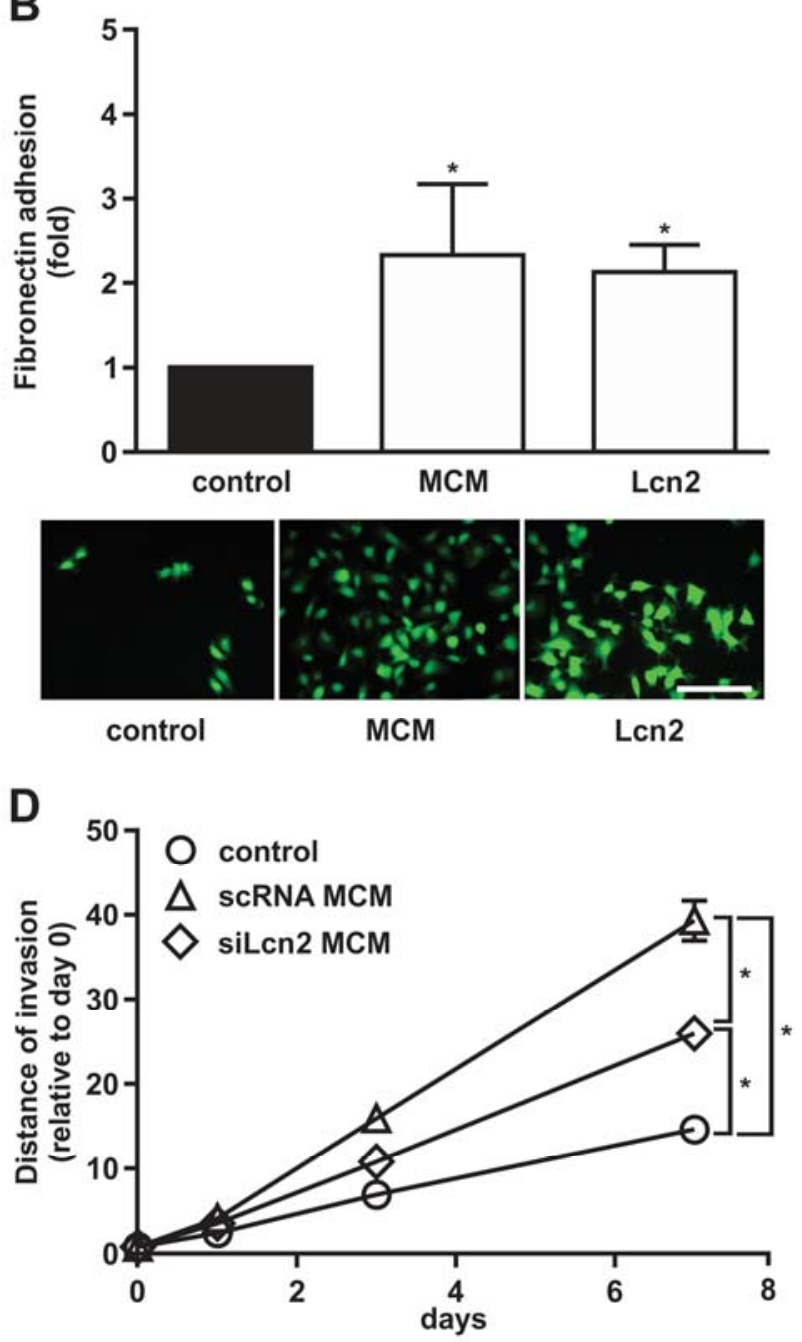

D

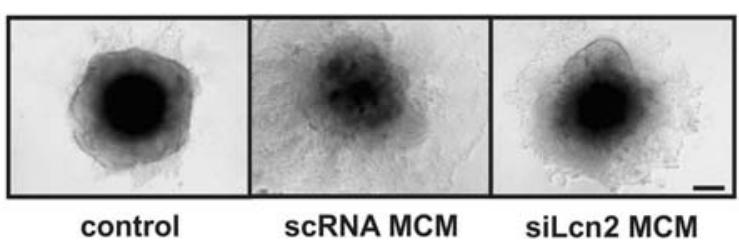

Fig. 2: Lcn2 induces the metastatic spread in vitro

(A-C) MCF-7 cells were stimulated with $1 \mu \mathrm{g} / \mathrm{ml}$ recombinant human Lcn2, macrophageconditioned medium (MCM), or remained unstimulated for 4 days. Adhesion of Cell Tracker green-labelled cells to (A) collagen I and to (B) fibronectin coated layers was quantified after $2 \mathrm{~h}$. Representative pictures of the adhesion assay are shown. Scale bar: $200 \mu \mathrm{m}$. (C) Transendothelial migration of pre-treated MCF-7 cells through a layer of HPMEC for $24 \mathrm{~h}$ was assessed by a Boyden chamber approach and quantified as the number of migrated cells. (D) MCF-7 spheroids were treated with scRNA MCM containing 
Lcn2, siLcn2 MCM deficient of Lcn2, or remained unstimulated (control) for 10 days and were embedded into a collagen I matrix for additional 7 days. The distance of spheroid cell invasion from the spheroid border into the matrix was measured after 1, 3 and, 7 days and is represented as distance of invasion relative to day 0 . Representative pictures are shown. Scale bar: $200 \mu \mathrm{m}$. Data are means \pm S.D., $n \geq 3,{ }^{*} p<0.05$ vs. controls, ${ }^{* *} p<0.01$ vs. controls, ${ }^{* * *} \mathrm{p}<0.001$ vs. controls.
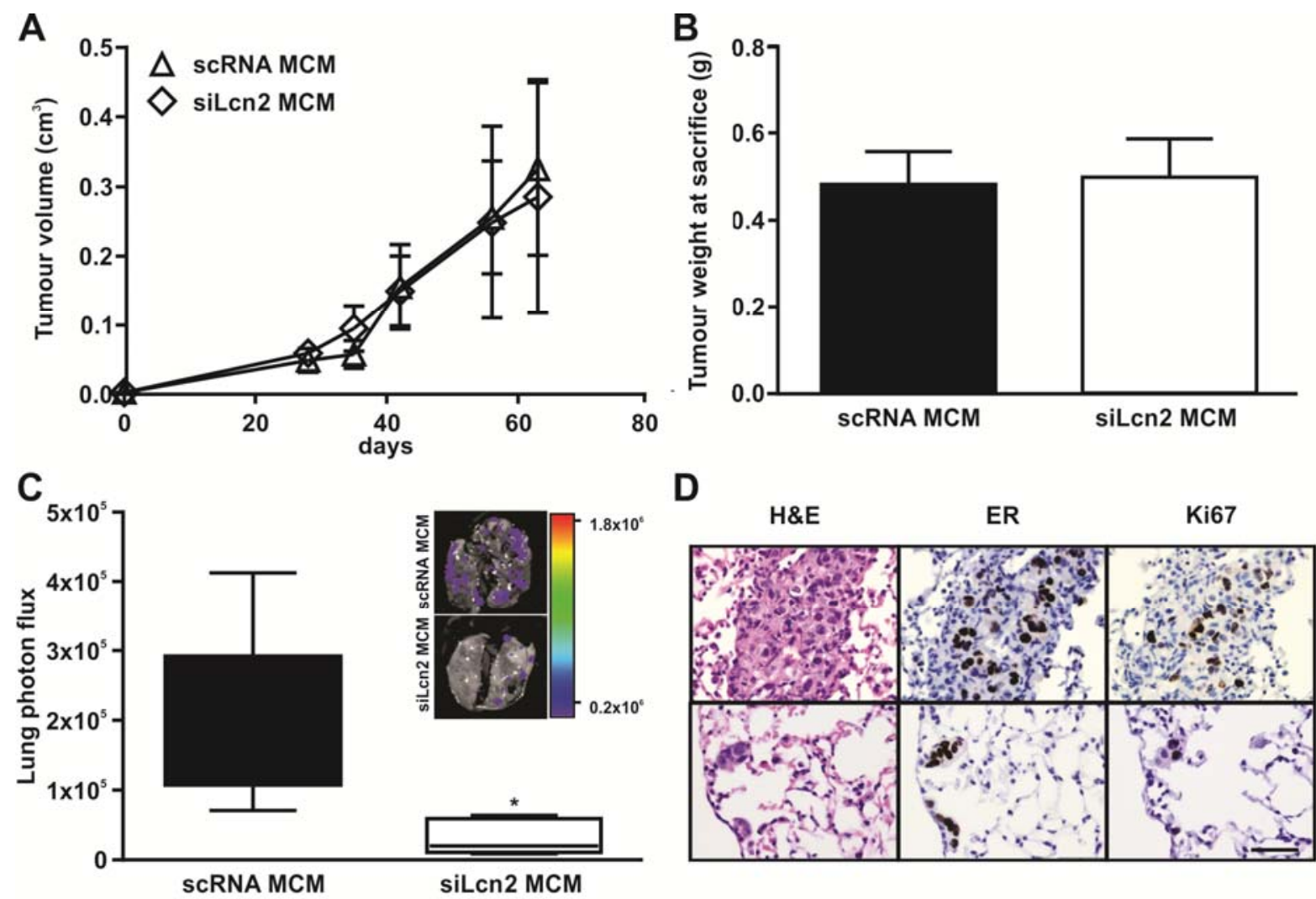

Fig. 3: TAM-released Lcn2 facilitates lung colonization in vivo

(A-D) MCF-7 cells were stimulated with scRNA MCM and siLcn2 MCM for 3 days. $3 \times 10^{6}$ tumour cells were implanted into the mammary fat pad of female BALB/c nude mice. (A) Tumour growth analysis was followed by IVIS until the tumour became palpable. (B) Tumour weight was calculated at sacrifice and (C) disseminated tumour cells were detected via the ex vivo photon flux measurement using the IVIS-200 system. (D) Fixed lungs were embedded in paraffin and the sections stained with H\&E to detect metastases. Furthermore, lung sections were stained for ER and Ki-67 by immunohistochemistry. 
Representative pictures of a macroscopic metastatic lesion (upper panel) and a microscopic lesion (lower panel) are shown. Scale bar: $50 \mu \mathrm{m}$. Data are means \pm S.D., $n \geq$ $3,{ }^{*} p<0.05$ vs. controls.

A

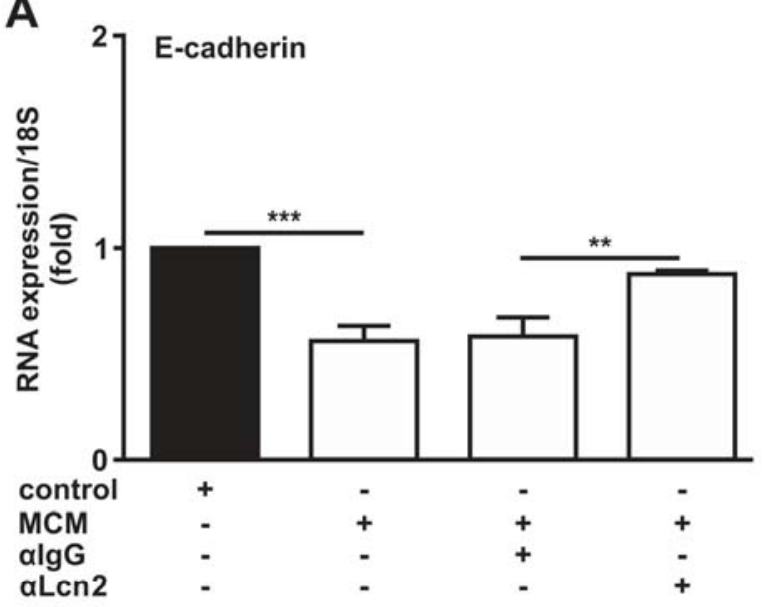

C

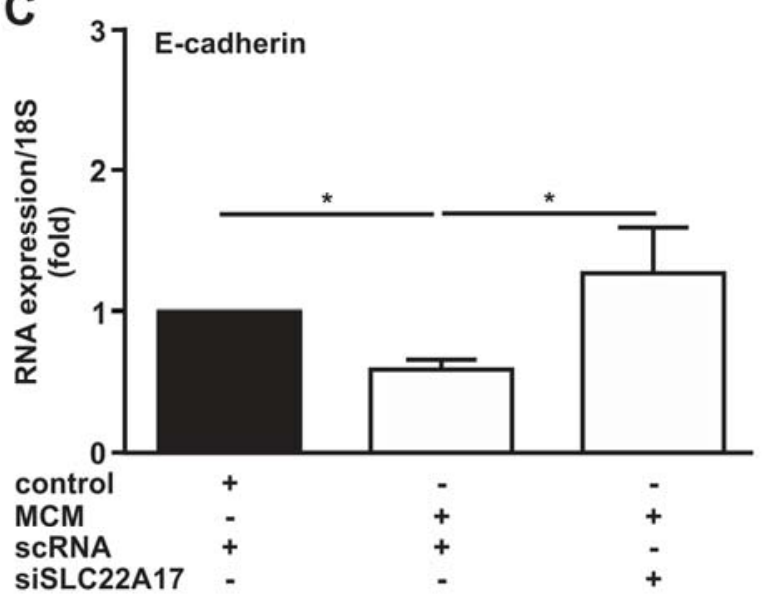

E

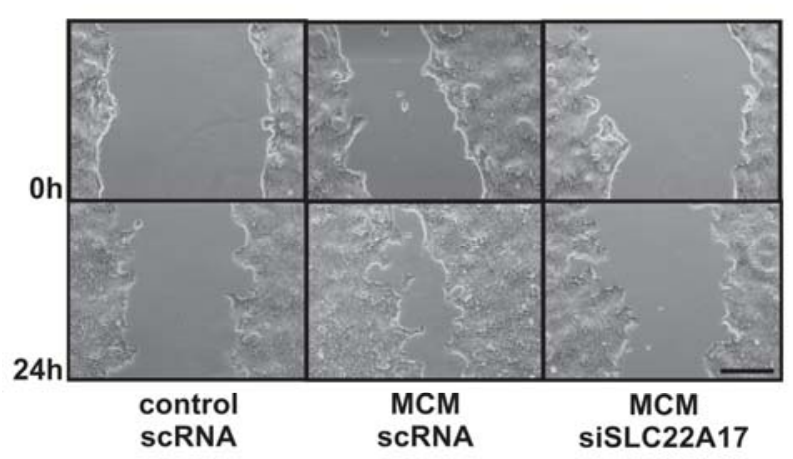

B

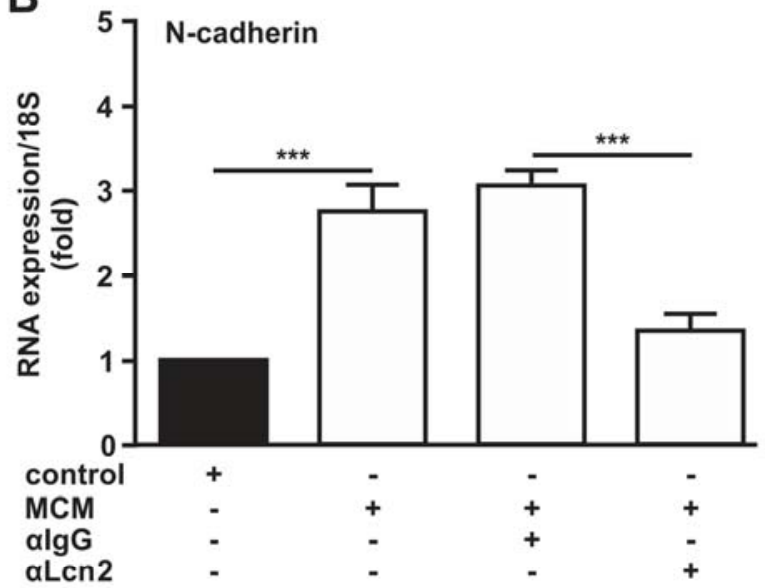

D

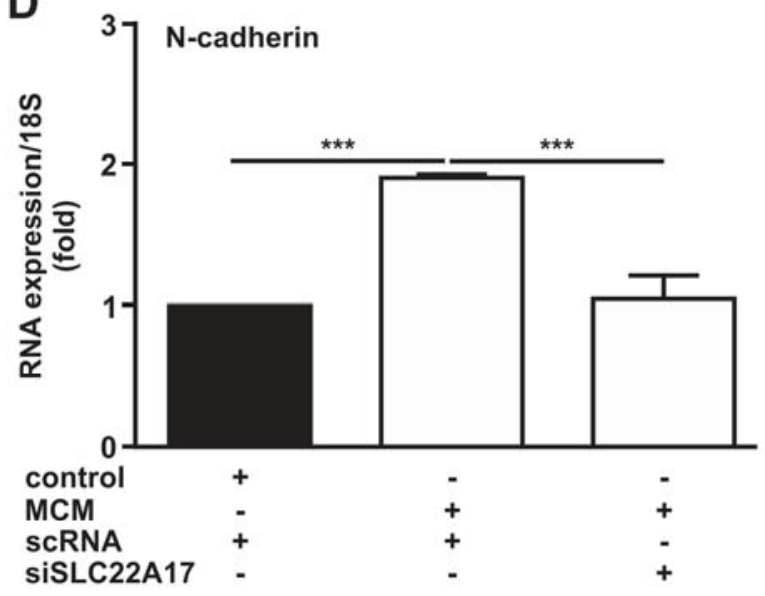

$\mathbf{F}$

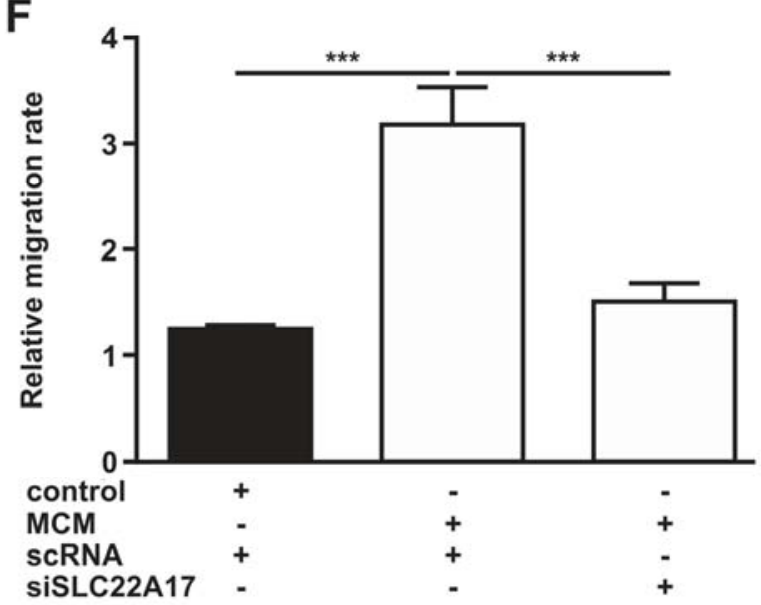

Fig. 4: Macrophage-derived Len2 induces EMT through its specific receptor

\section{SLC22A17}

This article is protected by copyright. All rights reserved. 
(A-B) MCF-7 cells were stimulated with MCM supplemented with a neutralizing antibody against Lcn2 ( $\alpha \mathrm{Lcn} 2$ ) or a control isotype matching $\lg$ antibody (algG) for $24 \mathrm{~h}$. Expression of mRNA encoding (A) E-cadherin $(C D H 1)$ and $(B) \mathrm{N}$-cadherin $(C D H 2)$ was measured by RT-qPCR. (C-D) MCF-7 cells were treated with either a scrambled control siRNA (scRNA) or siRNA to knockdown the Lcn2 receptor SLC22A17 (siSLC22A17) and stimulated with MCM for $24 \mathrm{~h}$. Expression of mRNA encoding (C) E-cadherin and (D) Ncadherin was assessed by RT-qPCR. (E-F) Cell migration assays were performed for MCM-treated MCF-7 cells previously transfected with scRNA or siSCL22A17. (E) Representative pictures and (F) quantification of migration after $24 \mathrm{~h}$. Scale bar: $100 \mu \mathrm{m}$. Data are means \pm S.D., $n \geq 3,{ }^{*} p<0.05$ vs. controls, ${ }^{* *} p<0.01$ vs. controls, ${ }^{* * *} p<0.001$ vs. controls. 

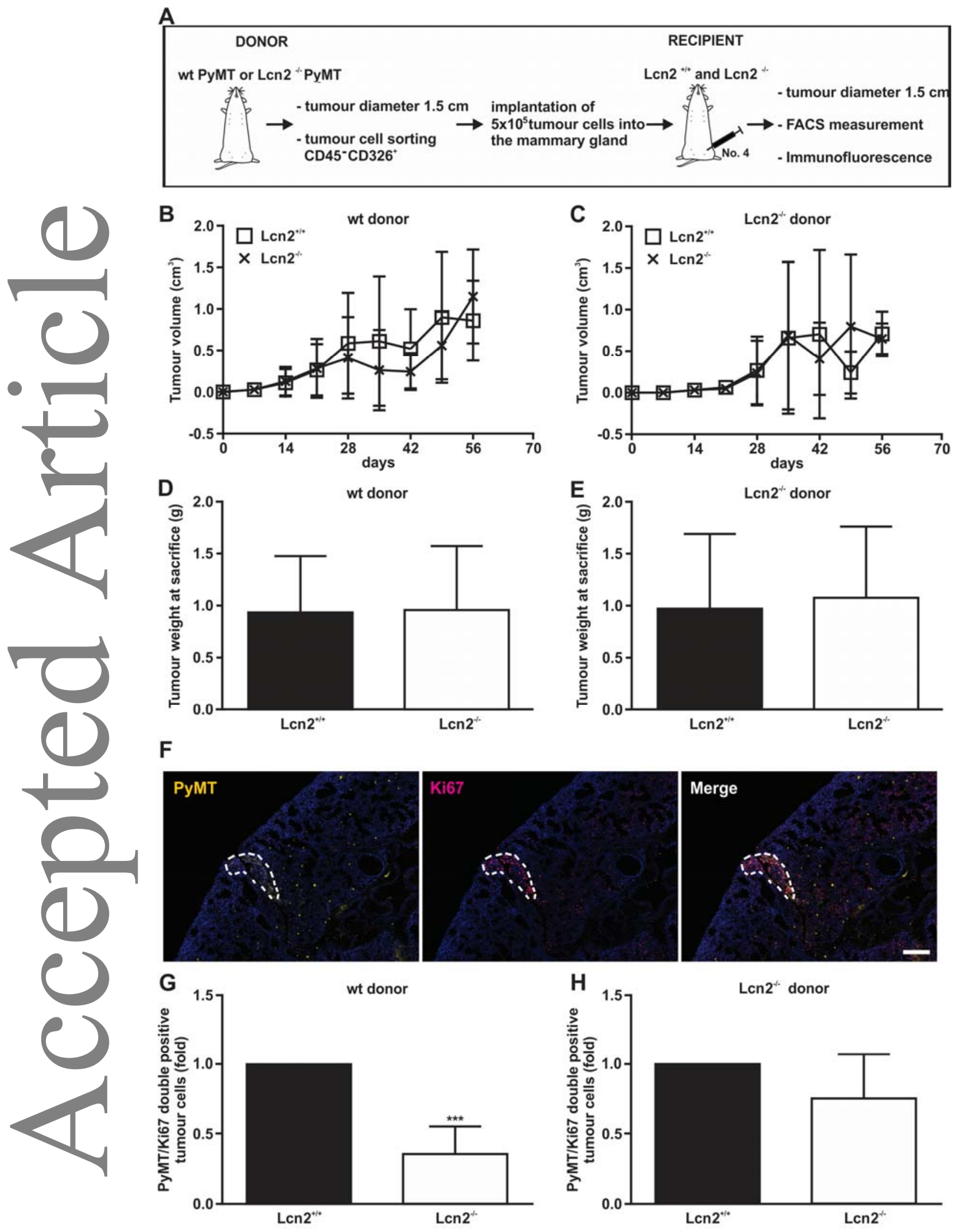

Fig. 5: Stroma-derived Len2 induces breast cancer cell dissemination into the lung

This article is protected by copyright. All rights reserved. 
(A) Wt PyMT and Lcn2 ${ }^{-/}$PyMT donors were sacrificed and CD45 $/$CD $326^{+}$tumour cells were FACS sorted. Tumour cells were implanted into the mammary gland of $\mathrm{Lcn}^{+/+}$and $\mathrm{Lcn}^{-/-}$recipient mice. (B-C) Tumour growth was detected by palpation of recipient mice and tumour volume was calculated as described in Methods. Analysis of the tumour volume in (B) wt PyMT donor cells in $\mathrm{Lcn}^{+/+}(\mathrm{n}=6)$ and $\mathrm{Lcn}^{-/-}(\mathrm{n}=6)$ recipient mice and (C) $\mathrm{Lcn}^{-/-}$donor cells in $\mathrm{Lcn}^{+/+}(\mathrm{n}=4)$ and $\mathrm{Lcn}^{-/-}(\mathrm{n}=4)$ recipient mice. (D-E) Recipient mice were sacrificed when the tumour developed a diameter of $1.5 \mathrm{~cm}$ and tumour weight was assessed ( $n \geq 4)$. (F) Lungs were co-stained for PyMT and Ki-67 by immunofluorescence. DAPI was used to detect nuclei. A PyMT- and Ki-67-double positive tumour cell lesion is encircled. Scale bar: $200 \mu \mathrm{m}$. (G-H) Quantification of PyMT- and Ki-67-double positive tumour cells. (G) Wt PyMT donor cells in Lcn2 ${ }^{+/+}(n=5)$ and $\mathrm{Lcn}^{-/-}(\mathrm{n}=5)$ recipient mice and (H) $\mathrm{Lcn}^{-{ }^{-}}$donor cells in $\mathrm{Lcn}^{+/+}(\mathrm{n}=3)$ and $\mathrm{Lcn}^{-{ }^{--}}(\mathrm{n}=3)$ recipient mice are displayed. Data are means \pm S.D., ${ }^{* * *} p<0.001$ vs. controls.

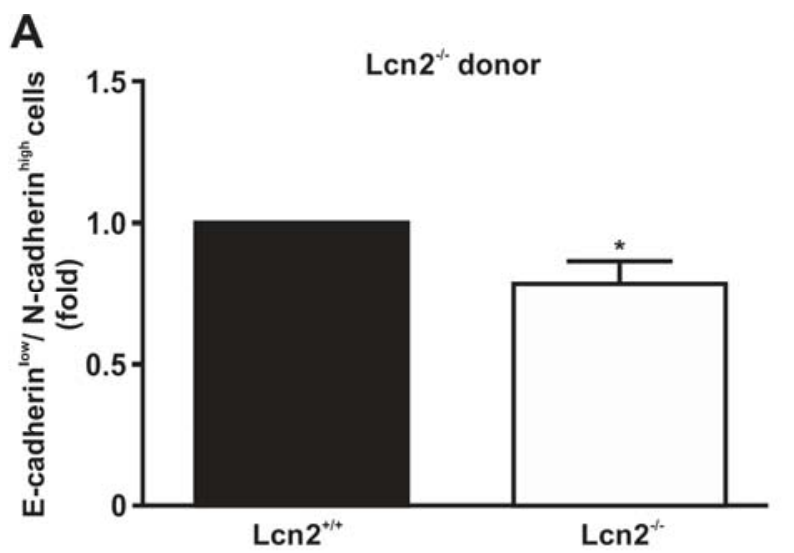

C

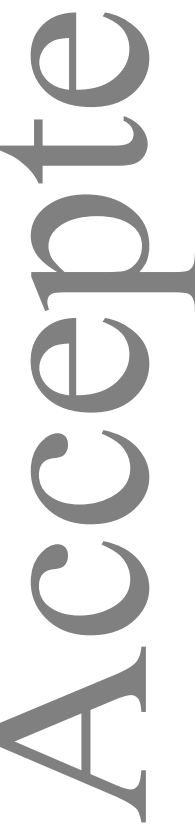

\section{B}
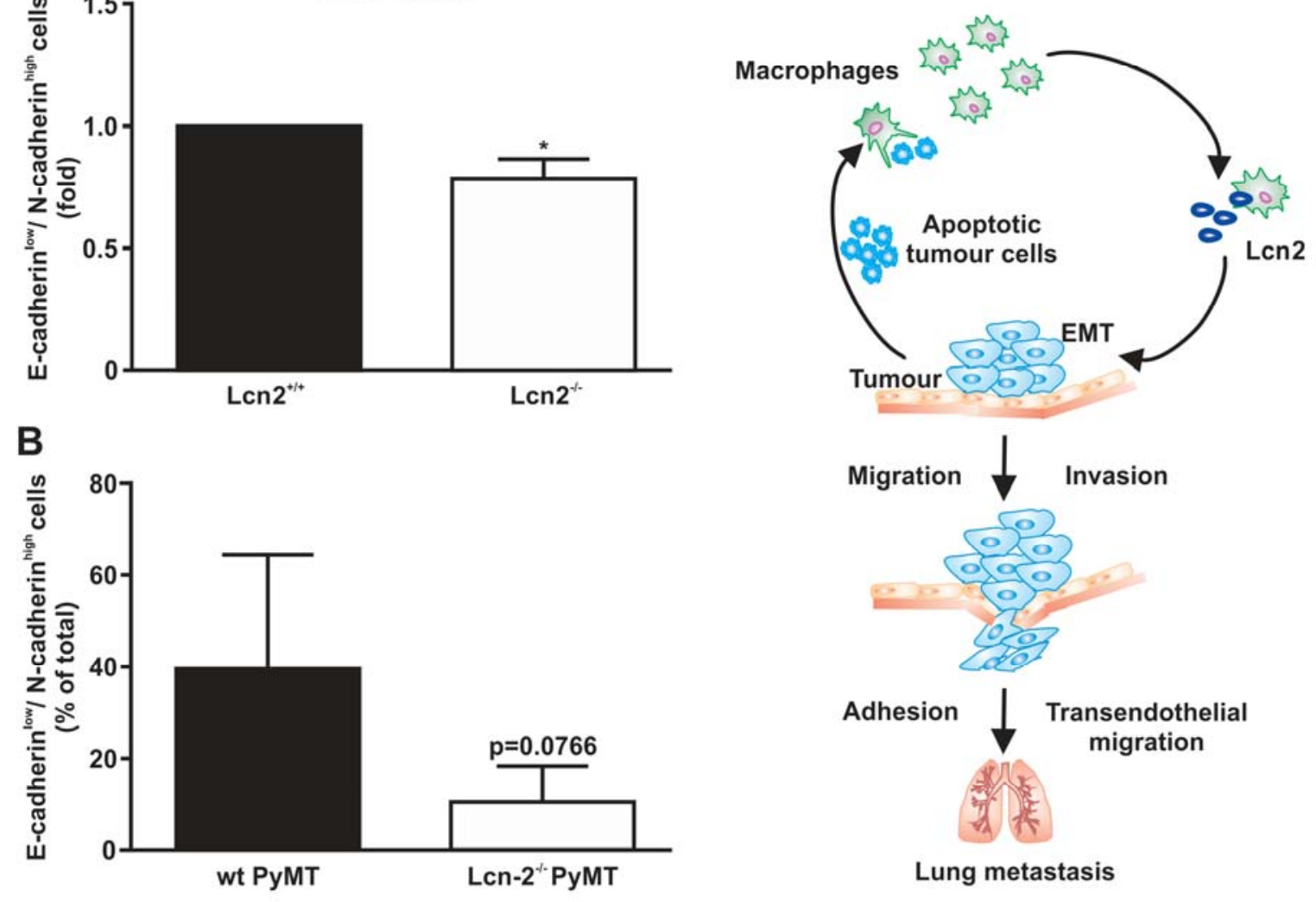

Lung metastasis

Fig. 6: Stroma-released Lcn2 fosters EMT

This article is protected by copyright. All rights reserved. 
(A) E-cadherin ${ }^{\text {low }} / \mathrm{N}$-cadherin ${ }^{\text {high }}$ cells were detected in the tumours of $\mathrm{Lcn}^{+/+}(\mathrm{n}=3)$ and $\mathrm{Lcn}^{-/-}(\mathrm{n}=3)$ recipient mice implanted with $\mathrm{Lcn} 2^{-/-}$PyMT donor cells using flow cytometry. (B) Detection of the E-cadherin ${ }^{\text {low }} / \mathrm{N}$-cadherin ${ }^{\text {high }}$ cells in the tumours of wt PyMT $(n=12)$ and $\mathrm{LCn}^{-/-}$PyMT donors $(n=3)$. Data are means \pm S.D., ${ }^{*} p<0.05$ vs. controls. (C) Schematic representation of the results.

This article is protected by copyright. All rights reserved. 